\title{
Coronary endothelial dysfunction after ischemia and reperfusion: a new therapeutic target?
}

K. Laude, C. Thuillez and V. Richard

\author{
IN SERM E9920, Faculté de M édecine-Pharmacie de Rouen, Rouen, France
}

\section{Correspondence \\ V. Richard \\ INSERM E9920 \\ Faculté de Médecine-Pharmacie \\ de Rouen \\ 22 Bd Gambetta \\ 76183 Rouen Cedex \\ France \\ Fax: + 33-2-3514-8365 \\ E-mail: vincent.richard@univ-rouen.fr \\ Presented at the XIV Annual Meeting of the Federação de Sociedades de Biologia Experimental, Caxambu, MG, Brazil, August 25-28, 1999.}

Received August 1, 2000 Accepted September 13, 2000

\section{Abstract}

Although cardiac ischemia is usually characterized as a disease of the myocyte, it is clear that the vasculature, and especially endothelial cells, is also a major target of this pathology. Indeed, using a rat model of ischemia/reperfusion, we were able to detect severe endothelial dysfunction (assessed as a decreased response to acetylcholine) after

\section{Key words}

- Coronary endothelium

- Ischemia/reperfusion

- Preconditioning acute or chronic reperfusion. Given the essential role of the endothelium in the regulation of vascular tone, as well as platelet and leukocyte function, such a severe dysfunction could lead to an increased risk of vasospasm, thrombosis and accelerated atherosclerosis. This dysfunction can be prevented by free radical scavengers and by exogenous nitric oxide. Endothelial dysfunction can also be prevented by preconditioning with brief periods of intermittent ischemia, thus extending to coronary endothelial cells the concept of endogenous protection previously described at the myocyte level. Experiments performed on cultured cells showed that the endothelial protection induced by free radical scavengers or by preconditioning was due to a lesser expression of endothelial adhesion molecules such as intercellular adhesion molecule-1, leading to a lesser adhesion of neutrophils to endothelial cells. Identification of the mechanisms of this protection may lead to the development of new strategies aimed at protecting the vasculature in ischemic heart diseases.

\section{Introduction}

Vascular endothelial cells play an essential role in the control of vascular tone, but also in the regulation of smooth muscle cell growth, as well as of platelet and leukocyte function. Numerous experimental and clinical data suggest that these essential physiological functions of the endothelium are altered in various pathophysiological situations, such as hypertension, hypercholesterolemia or diabetes. Such dysfunction can be characterized by an altered capacity of the endothelium to release nitric oxide (NO), associated with an increased production of oxygen-derived free radicals such as superoxide anions.

A similar impairment in the endothelial synthesis of NO has also been detected at the level of the coronary circulation after myocardial ischemia and acute or chronic reperfusion. Given the central role of $\mathrm{NO}$ as a vasodilating agent, but also as an inhibitor of platelet aggregation and leukocyte adhesion, 
it is likely that such a persistent impairment may have important deleterious consequences for the coronary arterial wall. Thus, coronary endothelial cells may be considered as a major therapeutic target of antiischemic treatments.

\section{Endothelium and myocardial ischemia}

Evidence that myocardial ischemia leads to coronary endothelial dysfunction was first obtained by $\mathrm{Ku}(1)$ who showed that a 90 min period of ischemia followed by 1-2-h reperfusion was associated with a decreased endothelium-dependent relaxation in response to thrombin in canine coronary arteries. These findings were rapidly extended to other endothelium-dependent vasodilators such as acetylcholine (2). The impaired endothelium-dependent responses were accompanied by marked structural injury to endothelial cells (assessed by electron microscopy). However, the response to nitroprusside, an endothelium-independent vasodilator, was intact.

Most of the experimental studies of endothelial dysfunction after ischemia/reperfusion involve in vivo coronary occlusion in large species (especially dogs and pigs) followed by studies of endothelium-dependent vasorelaxation in isolated coronary arteries. In a few studies, endothelial dysfunction is evaluated in isolated hearts submitted to ischemia in vitro. In our laboratory, we developed a rat model of myocardial infarction to assess coronary endothelial dysfunction following ischemia and reperfusion. This model consists of submitting Wistar rats to 20min ischemia followed by 60 -min reperfusion. At the end of this infarct protocol, hearts are removed and placed in physiological buffer for vascular study. Thus, coronary artery segments (1.5-2 mm long and 200$300 \mu \mathrm{m}$ in diameter) are taken distal to the site of occlusion and mounted on small vessel wire myographs to study their reactivity in the presence of vasoactive agents. This model allows us to focus on the consequences of ischemia/reperfusion at the level of large epicardial or medium-size intramyocardial coronary arteries.

The first results obtained with the use of this model were that ischemia/reperfusion decreased significantly the endothelium-dependent relaxation of the coronary artery segments in response to acetylcholine, compared with those of sham animals (3). However, no alteration in the relaxing response to acetylcholine appeared with the arteries taken from animals subjected to ischemia alone, in accordance with previous results (4), suggesting that the endothelial dysfunction observed with the myocardial ischemia is a manifestation of reperfusion injury. Moreover, the contractile responses to serotonin, which in this rat coronary preparation is not associated with the release of endotheliumderived relaxing factors, and thus only induces direct smooth muscle contraction (5), and the relaxing responses to SIN-1, an NO donor, were not modified by ischemia alone or followed by reperfusion.

Thus, myocardial ischemia/reperfusion is associated with an altered NO-dependent relaxation of coronary arteries. Additional experiments showed that incubation of the arterial segments with the substrate of NO synthase L-arginine could not reverse this impairment, in contrast to other diseases such as hypercholesterolemia. These results suggest that the impaired response to acetylcholine is mostly the consequence of the absence of structurally intact endothelial cells and not that of a selective defect in $\mathrm{NO}$ synthase activity, or a specific impairment of the transduction pathway linking muscarinic receptors to NO synthase. This hypothesis is in accordance with electron microscopy studies showing structural changes in the vascular wall (6).

Endothelial dysfunction after reperfusion is not a transient phenomenon, since it persists for at least 4-6 weeks in experimental 
models $(6,7)$. However, while acute dysfunction is the consequence of structural injury to the endothelial cells, chronic changes are rather a manifestation of dysfunctional regenerated endothelium, as previously demonstrated after balloon injury (8).

\section{Mechanisms of reperfusion-induced coronary endothelial dysfunction}

The observation that the reintroduction of molecular oxygen at reperfusion is required to produce postischemic endothelial dysfunction is consistent with the view that ischemia/reperfusion injury to the endothelium may result from the generation of reactive oxygen species. This has now been clearly demonstrated by the observation that endothelial dysfunction can be attenuated or prevented by scavengers of these species. In our rat model of myocardial ischemia/reperfusion, a continuous intravenous infusion of the free radical scavenger $N$-2-mercaptopropionyl glycine (MPG) during ischemia and reperfusion resulted in the prevention of the coronary endothelium-dependent relaxation in response to acetylcholine (9). There is also evidence in species other than rats that the reperfusion-induced endothelial injury is the consequence of the production of oxygen-derived free radicals because impaired endothelium-dependent relaxation can be restored by treatment with superoxide dismutase and catalase in both large coronary arteries $(4,10)$ and the microcirculation (11).

Several mechanisms may explain the endothelial injury induced by free radicals. Once produced, superoxide anions may directly inactivate NO $(12,13)$. Since NO is a potent inhibitor of neutrophil activation and adhesion (14), the decreased NO production may lead to the development of an acute inflammatory response. Moreover, since free radicals produced during reperfusion also trigger the rapid adhesion of neutrophils to endothelial cells through the induction of adhesion molecules such as selectins or intercellular adhesion molecule-1 (ICAM-1) (15), the combined effects of the decreased production of $\mathrm{NO}$ and the increased production of free radicals will reinforce the adhesion of neutrophils to the endothelial cells, setting the stage for an amplification of the neutrophil-mediated endothelial injury.

\section{Protection of coronary endothelial cells}

Prolonged ischemia and reperfusion are responsible for a cascade of reactions leading to endothelial injury characterized by a decrease in NO production. Given the important vasodilator property of $\mathrm{NO}$, such an impairment may lead to an increased coronary vasoconstriction and an increased risk of vasospasm. Moreover, endothelial dysfunction after reperfusion may favor platelet aggregation and thus increase the risk of thrombosis. Taken together, those observations indicate that the prevention of endothelial dysfunction/injury is an important therapeutic goal. However, little is known about the endothelial effects of most of the known anti-ischemic interventions.

One of the most potent anti-ischemic interventions known to date is the concept of endogenic protection of ischemic myocardium described by Murry et al. (16), and termed "preconditioning". According to these experiments, submitting the heart to short episodes of ischemia separated by intermittent reperfusion renders the heart more resistant to prolonged ischemia and markedly limits infarct size. Preconditioning also confers protection against the severe ventricular rhythm disturbances that occur during subsequent ischemia and reperfusion $(17,18)$.

Using our rat model of myocardial ischemia/reperfusion in vivo, we demonstrated that such a protection can be obtained at the level of the coronary endothelial cells (3). Indeed, 3 cycles of 5-min ischemia/5-min reperfusion prevented the impaired endothe- 
lium-dependent relaxing responses to acetylcholine when the artery was subjected to immediate prolonged ischemia/reperfusion (Figure 1). Moreover, we demonstrated that this was not a transient phenomenon and that the protective effect can be prolonged at least 4 weeks after reperfusion (6).

The mechanisms of the endothelial protection induced by preconditioning are still not well established. Given the central role of neutrophil adhesion in endothelial injury during reperfusion, we hypothesized that the protective effect of preconditioning is due in part to a decreased production of endothelial adhesion molecules leading to a lesser adhesion of neutrophils to endothelium. Indeed, as mentioned above, reperfusion injury to the endothelium is mediated by neutrophils which adhere via the expression of adhesion molecules such as ICAM-1 $(19,20)$. To test this hypothesis, we subjected cultured rat aortic endothelial cells to in vitro anoxia and reoxygenation. In this context, we found that preconditioning abolished the increased

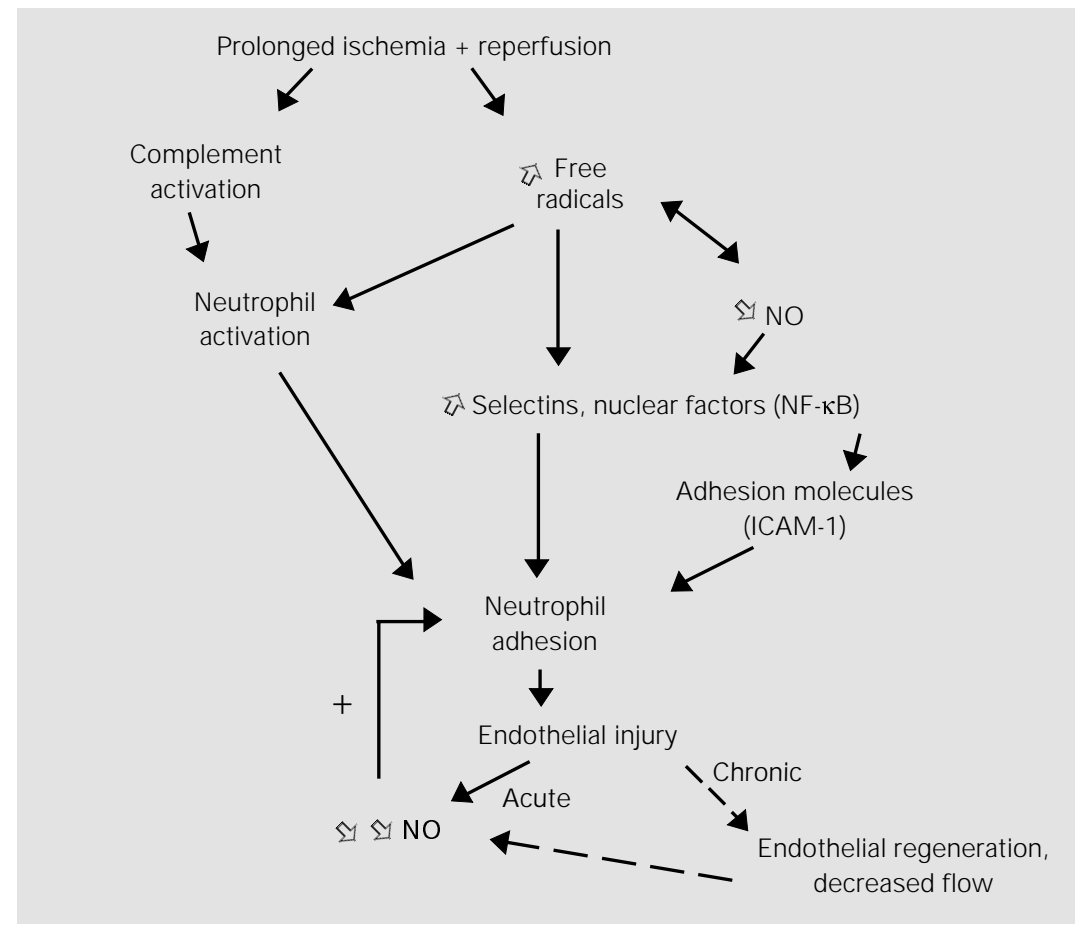

Figure 1 - Possible mechanisms of endothelial injury after ischemia/reperfusion. NO, Nitric oxide; ICAM-1, intercellular adhesion molecule-1.
ICAM-1 expression and reduced the adhesion of neutrophils observed with anoxiareoxygenation alone (21). These protective effects of preconditioning can be mimicked by the free radical scavenger MPG. Moreover, incubation of endothelial cells with MPG, chelerythrin (an inhibitor of protein kinase C) or $N^{\mathrm{G}}$-nitro-L-arginine (an inhibitor of NO synthases) during preconditioning abolished its protective effects on ICAM-1 expression and neutrophil adhesion, suggesting a role for protein kinase $\mathrm{C}$, free radicals and $\mathrm{NO}$ as triggers of preconditioning in this model.

Although preconditioning the heart has efficient protective effects, the protection induced by this intervention is transient, and disappears if the period of reperfusion separating preconditioning from prolonged ischemia extends over $2-3 \mathrm{~h}$. This time course possibly limits its therapeutic application. However, recent evidence showed that myocardial protection reappears several hours after preconditioning. Indeed, preconditioning performed $24 \mathrm{~h}$ before prolonged coronary occlusion is associated with a significant limitation of infarct size in dogs (22) or in rabbits (23), and extends to other aspects of ischemia/reperfusion injury such as myocardial stunning (24). To assess whether such a "second window" of protection exists at the level of coronary endothelial cells, we subjected rats to 3 periods of intermittent left coronary artery occlusion and allowed them to recover. Twenty-four hours later, rats were re-anesthetized and subjected to the standard ischemia/reperfusion protocol described above (9). Those experiments showed that, in addition to having beneficial effects on infarct size and postischemic myocardial contractile dysfunction, delayed preconditioning also protects coronary endothelial cells against ischemia and reperfusion injury (9). Moreover we found that the beneficial effect of delayed preconditioning on endothelial protection was abolished by administration of MPG (9). These results suggest a 
triggering role of free radicals in delayed preconditioning, which may act through stimulation of the expression of various protective proteins, including NO synthases and antioxidant enzymes (Figure 2). Similar results were obtained about infarct size (25), as well as myocardial stunning (26).

Again, the mechanisms of such delayed preconditioning were evaluated in cultured endothelial cells; as observed with "classic" preconditioning, we found that delayed preconditioning was associated with a decrease in neutrophil adhesion and a complete abolition of ICAM-1 expression (27). These changes in endothelium-neutrophil interactions were accompanied by an increased expression of several antioxidant enzymes and of NO synthases.

Among the different possible triggers and mediators of delayed preconditioning, $\mathrm{NO}$ seems to play a dominant role. Indeed, it has been demonstrated that brief ischemia was associated with a delayed increase in the coronary flow response to acetylcholine and bradykinin (28). Moreover, numerous studies have shown that NO plays a central role in the delayed protective effects of preconditioning in myocardial stunning (29) and infarction $(30,31)$ via the inducible nitric oxide synthase (iNOS). Thus, we tested whether a similar involvement of iNOS could be observed at the level of the endothelial cells. For this purpose, we administered the selective iNOS inhibitor, $N$-(3-(aminomethyl) benzyl)acetaminide (1400 W) to preconditioned rats (32). Although $1400 \mathrm{~W}$ indeed blocked iNOS activity in vivo under those experimental conditions, it did not affect the endothelial protection induced by delayed preconditioning. Thus, in contrast to the myocytes, iNOS is not involved as a mediator of delayed preconditioning in endothelial cells. This opposite effect of iNOS inhibition may be explained in part by the distinct effect of NO on cardiomyocytes and endothelial cells. Indeed, iNOS activation by inflammatory stimuli produces high concen- trations of $\mathrm{NO}$ (33). In cardiomyocytes, such high levels of NO probably induce a decrease in metabolic requirements and oxygen consumption, and this effect is probably responsible for the anti-ischemic role of iNOS. Contrary to cardiac myocytes, an overproduction of NO in endothelial cells may have deleterious consequences, in part by its reaction with oxygen-derived free radicals to produce highly reactive intermediates such as peroxynitrites. In this context, NO may act as a pro-inflammatory stimulus.

Our experiments rule out the hypothesis of a role of iNOS in the endothelial effects of delayed preconditioning, but do not exclude a role for NO produced by other enzymes such as endothelial NO synthase (eNOS). Indeed, endogenous stimulation of eNOS (for example by acetylcholine) may exert marked endothelial protective effects after ischemia and reperfusion (34). Moreover, our experiments on cultured cells demonstrated an increase of the nuclear factor AP1 in the endothelial cell nucleus after preconditioning (27), and this may be correlated to the increased level of eNOS mRNA, since AP-1 is responsible for the enhanced

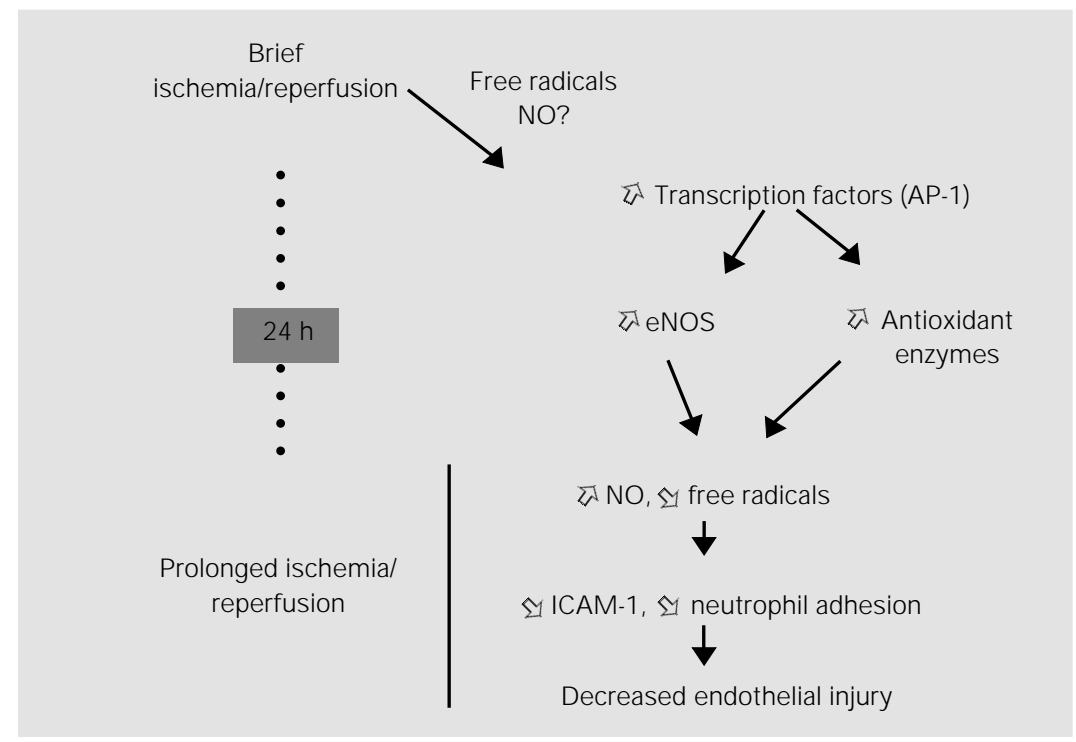

Figure 2 - Potential mechanisms of the endothelial protective effects of late preconditioning. NO, Nitric oxide; eNOS, endothelial nitric oxide synthase; ICAM-1, intercellular adhesion molecule-1. 
expression and activity of eNOS (35). Whether changes in eNOS contribute to the endothelial effects of preconditioning is currently being investigated in our laboratory.

Coronary endothelial dysfunction is an important, although long overlooked, component of cardiac ischemia/reperfusion injury. Such endothelial dysfunction seems to be mainly the consequence of reperfusioninduced production of free radicals and ad- hesion of neutrophils. However, identification of protective pathways against reperfusion-induced endothelial injury, including preconditioning, may lead to the discovery of potent interventions aimed at limiting vascular injury after ischemia, with possible marked beneficial effects on the incidence of vasospasm, thrombosis and further atherosclerosis.

\section{References}

1. Ku DD (1982). Coronary vascular reactivity after acute myocardial ischemia. Science, 218: 576-578.

2. Van Benthuysen KM, McMurtry IF \& Horwitz LD (1987). Reperfusion after acute coronary occlusion in dogs impairs endothelium-dependent relaxation to acetylcholine and augments contractile reactivity in vitro. J ournal of Clinical Investigation, 79: 265-274.

3. Richard V, Kaeffer N, Tron C \& Thuillez C (1994). Ischemic preconditioning protects against coronary endothelial dysfunction induced by ischemia and reperfusion. Circulation, 89: 1254-1261.

4. Tsao PS, Aoki N, Lefer DJ , J ohnson III G $\&$ Lefer AM (1990). Time course of endothelium dysfunction and myocardial injury during myocardial ischemia and reperfusion in the cat. Circulation, 82: 1402-1412.

5. Nyborg NCB \& Mikkensen EO (1990). 5Hydroxytryptamine does not induce release of endothelium-derived relaxing factor in rat coronary arteries. European J ournal of Pharmacology, 186: 295-300.

6. Kaeffer N, Richard V, François A, Lallemand F, HenryJ P \& Thuillez C (1996). Preconditioning prevents chronic reperfusion-induced coronary endothelial dysfunction in rats. American J ournal of Physiology, 271: H842-H849.

7. Pearson PJ, Schaff HV \& Vanhoutte PM (1990). Long-term impairment of endothelium-dependent relaxations to aggregating platelets following reperfusion injury in canine coronary arteries. Circulation Research, 81: 1921-1927.

8. Shimokawa H, Aarhus LL \& Vanhoutte PM (1987). Porcine coronary arteries with regenerated endothelium have a reduced endothelium-dependent responsiveness to aggregating platelets and serotonin.
Circulation Research, 61: 256-270.

9. Kaeffer N, Richard V \& Thuillez C (1997). Delayed coronary endothelial protection 24 hours after preconditioning. Role of free radicals. Circulation, 96: 2311-2316.

10. Gross GJ , O'Rourke ST \& Pelc LR (1992). Myocardial and endothelial dysfunction after multiple, brief coronary occlusions: role of oxygen radicals. American J ournal of Physiology, 263: H1703-H1709.

11. Mehta J L, Nichols WW, Donnelly WH, Lawson DL, Thompson L, Rietter M \& Saldeen TGP (1989). Protection by superoxide dismutase from myocardial dysfunction and attenuation of vasodilator reserve after coronary occlusion and reperfusion in dog. Circulation Research, 65: 1283-1295.

12. Rubanyi GM \& Vanhoutte PM (1986). Superoxide anions and hyperoxia inactivate endothelium-derived relaxing factor. American J ournal of Physiology, 250: H822$\mathrm{H} 827$.

13. Gryglewski RJ , Palmer RMJ \& Moncada S (1986). Superoxide anion is involved in the breakdown of endothelium-derived relaxing factor. Nature, 320: 454-460.

14. Kubes P, Suzuki M \& Granger DN (1991). Nitric oxide: an endogenous modulator of leukocyte adhesion. Proceedings of the National Academy of Sciences, USA, 88: 4651-4655

15. Thiagarajan RR, Winn RK \& Harlan J M (1997). The role of leukocyte and endothelial adhesion molecules in ischemiareperfusion injury. Thrombosis and Haemostasis, 78: 310-314.

16. Murry $C E$, J ennings $R B \&$ Reimer $K A$ (1986). Preconditioning with ischemia: a delay of lethal cell injury in ischemic myocardium. Circulation, 74: 1124-1136.

17. Lawson CS \& Hearse DJ (1994). Ischemic preconditioning against arrhythmias: an anti-arrhythmic or an anti-ischemic phenomenon? Annals of the New York Academy of Sciences, 723: 138-157.

18. Parratt J R, Vegh A, Kaszala K \& Papp J G (1996). Protection by preconditioning and cardiac pacing against ventricular arrhythmias resulting from ischemia and reperfusion. Annals of the New York Academy of Sciences, 793: 98-107.

19. Kukielka GL, Hawkins HK, Michael L, Manning AM, Youker K, Lane C, Entman ML, Wayne Smith C \& Anderson DC (1993). Regulation of intercellular adhesion molecule-1 (ICAM-1) in ischemic and reperfused myocardium. J ournal of Clinical Investigation, 92: 1504-1516.

20. Youker KA, Hawkins HK, Kukielka GL, Perrard J L, Michael LH, Ballantyne CM, Smith CW \& Entman ML (1994). Molecular evidence for induction of intracellular adhesion molecule- 1 in the viable border zone associated with ischemia-reperfusion injury of the dog heart. Circulation, 89: 2736-2746.

21. Beauchamp P, Richard V, Tamion F, Lallemand $F$, Lebreton J $P$, Vaudry $H$, Daveau M \& Thuillez C (1999). Protective effects of preconditioning in cultured rat endothelial cells. Effects of neutrophil adhesion and expression of ICAM-1 after anoxia and reoxygenation. Circulation, 100: 541-546.

22. Kuzuya T, Hoshida S, Yamashita N, Fuji H, Oe H, Hori M, Kamada T \& Tada M (1993). Delayed effects of sublethal ischemia on the acquisition of tolerance to ischemia. Circulation Research, 72: 1293-1299.

23. Marber MS, Latchman DS, Walker J M \& Yellon DM (1993). Cardiac stress protein elevation 24 hours after brief ischemia or heat stress is associated with resistance 
to myocardial infarction. Circulation, 88: 1264-1272.

24. Sun J Z, Tang XL, Knowlton AA, Park SW, Qiu Y \& Bolli R (1995). Late preconditioning against myocardial stunning: an endogenous protective mechanism that confers resistance to postischemic dysfunction 24 hours after brief ischemia in conscious pigs. J ournal of Clinical Investigation, 95: 388-403.

25. Yamashita N, Hoshida S, Taniguchi N, Kuzuya T \& Hori M (1998). A "second window of protection" occurs 24 hours after ischemic preconditioning in the rat heart. J ournal of Molecular and Cellular Cardiology, 30: 1181-1189.

26. Sun J Z, Tang XL, Park SW, Qiu Y, Turrens J F \& Bolli R (1996). Evidence for an essential role of reactive oxygen species in the genesis of late preconditioning against myocardial stunning in conscious pigs. J ournal of Clinical Investigation, 97: 562576.

27. Beauchamp P (1999). Effets protecteurs du préconditionnement de cellules endothéliales de rat en culture. Effets sur l'adhésion des neutrophiles et sur l'expression d'ICAM-1 après anoxie et réoxygéna- tion. Doctoral thesis, Université de Rouen, Rouen, France.

28. Kim SJ , Ghaleh B, Kudej RK, Huang CH, Hintze TH \& Vatner SF (1997). Delayed enhanced nitric oxide-mediated coronary vasodilation following brief ischemia and prolonged reperfusion in conscious dogs. Circulation Research, 81: 53-59.

29. Bolli $R, M$ anchikalapudi $S$, Tang $X L$, Takano H, Qiu Y, Guo Y, Zhang Q \& J adoon AK (1997). The protective effect of late preconditioning against myocardial stunning in conscious rabbit is mediated by nitric oxide synthase. Evidence that nitric oxide acts both as a trigger and as a mediator of the late phase of ischemic preconditioning. Circulation Research, 81: 1094-1107.

30. Imagawa J, Yellon DM \& Baxter GF (1999). Pharmacological evidence that inducible nitric oxide synthase is a mediator of delayed preconditioning. British J ournal of Pharmacology, 126: 701-708.

31. Guo $Y$, J ones WK, Xuan YT, Tang XL, Bao W, Wu WJ, Han $\mathrm{H}$, Laubach VE, Ping $\mathrm{P}$, Yang Z, Qiu Y \& Bolli R (1999). The late phase of ischemic preconditioning is abrogated by targeted disruption of the in- ducible NO synthase gene. Proceedings of the National Academy of Sciences, USA, 96: 11507-11512.

32. Laude K, Richard V, Henry J P, Lallemand F \& Thuillez C (2000). Evidence against a role of inducible nitric oxide synthase in the endothelial protective effects of delayed preconditioning. British J ournal of Pharmacology (in press).

33. Stoclet J C, Muller B, Andiantsitohaina R $\&$ Kleschyov A (1998). Overproduction of nitric oxide in the pathophysiology of blood vessels. Biochemistry, 63: 826-832.

34. Richard V, Blanc T, Kaeffer N, Tron C \& Thuillez C (1995). Myocardial and coronary endothelial protective effects of acetylcholine after myocardial ischaemia and reperfusion in rats: role of nitric oxide. British J ournal of Pharmacology, 115: 1532-1538.

35. Lopez-Ongil S, Hernandez-Perera O, Navarro-Antolin J , Perez DL, RodriguezPuyol M, Lamas S \& Rodriguez-Puyol D (1998). Role of reactive oxygen species in the signalling cascade of cyclosporine Amediated up-regulation of eNOS in vascular endothelial cells. British J ournal of Pharmacology, 124: 447-454. 